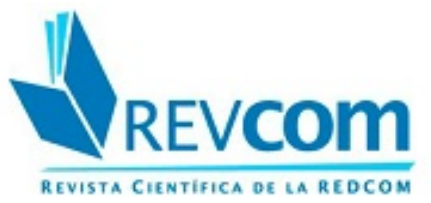

REVCOM. Revista científica de la red de carreras de Comunicación Social

ISSN: 2451-7836

redcom.revcom@gmail.com

Universidad Nacional de La Plata

Argentina

\title{
La segunda reforma universitaria: políticas de género y transversalización en las universidades
}

Torlucci, Sandra; Vazquez Laba, Vanesa; Pérez Tort, Mailén

La segunda reforma universitaria: políticas de género y transversalización en las universidades

REVCOM. Revista científica de la red de carreras de Comunicación Social, núm. 9, 2019

Universidad Nacional de La Plata, Argentina

DOI: https://doi.org/10.24215/24517836e016 


\section{La segunda reforma universitaria: políticas de género y transversalización en las universidades}

A segunda reforma da universidade: políticas de gênero e integração nas universidades.

Sandra Torlucci

DOI: https://doi.org/10.24215/24517836e016

Universidad Nacional de las Artes, Argentina

Vanesa Vazquez Laba

CONICET-IDAES/Universidad Nacional de San Martín, Argentina

Recepción: 10 Octubre 2019

Mailén Pérez Tort

IDAES/Universidad Nacional de San Martin, Argentina

Aprobación: 18 Octubre 2019

Recepción: 10 Octubre 2019

Aprobación: 18 Octubre 2019

\section{RESUMEN:}

En este artículo nos proponemos reconstruir el proceso de instauración de la agenda feminista en las instituciones universitarias de los últimos cinco años, a través del trabajo político de las feministas universitarias en articulación con las redes del movimiento de mujeres y feminista a nivel regional y nacional. A partir de la aprobación de los protocolos de actuación frente a las situaciones de discriminación y violencia de género sumado a la transversalización del enfoque de género en las políticas institucionales y en las actividades sustantivas de investigación, formación y extensión, así como también la sanción de la Ley 27.499 Ley Micaela y la adhesión del Consejo Interuniversitario Nacional (CIN) a la misma. Un hito fundamental de éste proceso es la creación en el año 2015 de la Red Interuniversitaria por la Igualdad de Género y contra las Violencias y en 2018 su posterior institucionalización en el CIN como RUGE, su nombre abreviado. Las líneas de acción de la RUGE se enfocaron en el fortalecimiento y elaboración de los protocolos de actuación, en la institucionalización de los espacios de género, la transversalizacion de la perspectiva de género en el sistema universitario, las capacitaciones establecidas por la Ley Micaela y la realización de un relevamiento del estado de situación de las políticas de género en las instituciones universitarias. En este artículo presentamos los resultados alcanzados logrando obtener una fotografía del sistema universitario en políticas de género.

Palabras ClaVe: Instituciones universitarias, políticas de género, transversalización, Ley Micaela.

\section{Resumo:}

Neste artigo, propomos reconstruir o processo de estabelecimento da agenda feminista nas instituições universitárias dos últimos cinco anos, através do trabalho político das feministas universitárias em articulação com as redes do movimento feminista e feminista nos níveis regional e nacional. Com base na aprovação dos protocolos de ação diante de situações de discriminação e violência de gênero, juntamente com a incorporação da abordagem de gênero nas políticas institucionais e nas atividades substantivas de pesquisa, treinamento e extensão, bem como na sanção de Lei 27.499 Lei Micaela e a adesão do Conselho Nacional da Interuniversidade (CIN) a ela. Um marco fundamental desse processo é a criação em 2015 da Rede Interuniversitária de Igualdade de Gênero e Contra a Violência e em 2018 sua subsequente institucionalização no CIN como RUGE, seu nome abreviado. As linhas de ação da RUGE se concentraram no fortalecimento e elaboração de protocolos de ação, na institucionalização dos espaços de gênero, na incorporação da perspectiva de gênero no sistema universitário, na capacitação estabelecida pela Lei Micaela e na realização de um pesquisa do estado da situação das políticas de gênero nas instituições universitárias. Neste artigo, apresentamos os resultados obtidos com a obtenção de uma fotografia do sistema universitário nas políticas de gênero.

Palavras-chave: Instituições universitárias, políticas de gênero, Lei Micaela. 


\section{INTRODUCCIÓN}

El proceso que vienen llevando a cabo las instituciones educativas es inédito en nuestro país. Si bien data desde principios del siglo pasado el involucramiento de las universitarias en la problemática de las mujeres, en los últimos tiempos hemos asistido a la instauración de las políticas de género en las instituciones como nunca antes se había dado. La fundación de los estudios de las mujeres y de género con la creación de los centros de estudios, institutos y programas de investigación de finales de los ' 80 y de la década de los ' 90 generaron, asimismo, las condiciones para que desde el año 2014 se comience un proceso de profundización y ensanchamiento de las políticas de género en las universidades. A partir de la aprobación de los protocolos de actuación frente a las situaciones de discriminación y violencia de género sumado a la transversalización del enfoque de género en las políticas institucionales y en las actividades sustantivas de investigación, formación y extensión se puede hablar, como lo sostiene la historiadora Ana Laura Martín de una segunda refundación de los estudios feministas en las universidades o "la gran reforma" como lo definió en una artículo la periodista Marta Vassallo.

Un hito de este proceso ha sido el lanzamiento en el año 2015 de la Red Interuniversitaria por la Igualdad de Género y contra las Violencias en la Universidad Nacional de San Martín con la participación de más de 20 universidades, facultades e institutos. La misma de definió originalmente como un espacio de promoción de actividades académicas, experiencias de gestión e intervención, así como, también, de circulación de ideas y propuestas para la transversalización de la perspectiva de género en el sistema universitario, proceso que ya se venía dando de manera dispersa en algunas universidades por la militancia de las universitarias. En el 2018, la Red se incorpora como organización en el marco del Consejo Interuniversitario Nacional (CIN), desde donde se propone colaborar en el diseño y desarrollo de políticas que contribuyan a erradicar las desigualdades de género y las violencias en todo el sistema universitario. Con las siglas "RUGE" como nombre abreviado, se designan las autoridades para el primer período 2018-2020, quedando como coordinadora Sandra Torlucci, Rectora de la Universidad Nacional de las Artes. ${ }^{1}$

De las asambleas y reuniones se delinearon diferentes propuestas de acción institucional basadas fundamentalmente en la creación y profundización de los protocolos y espacios de intervención de los casos de violencia de género; en la transversalización de la perspectiva de género en la formación y en investigación; y, también, en la construcción de planes/políticas de igualdad. Dentro de las actividades que viene llevando a cabo la RUGE se destacan dos muy importantes: por un lado, las "clínicas de casos", que son aquellos espacios donde se intercambian experiencias y se discuten modelos de atención y medidas institucionales para el acompañamiento de los casos de violencia de género; y, por otro lado, la organización de las capacitaciones a las autoridades y diferentes claustros en el marco de la "Ley Micaela" aprobada por el CIN este año. ${ }^{2}$

Por último, y es lo que presentamos y analizamos en este artículo, se realizó durante los meses de septiembre y octubre un relevamiento de las políticas de género en el sistema universitario. Dicho estudio de carácter cuantitativo, levantó datos de 61 encuestas auto administradas de forma online a las representantes titulares o suplentes ${ }^{3}$ de las universidades ante la RUGE $^{4}$. Los resultados alcanzados nos permiten obtener una fotografía del sistema universitario en políticas de género hasta el momento.

\section{Primer PASO: Protocolos de ACTUACión EN LAS UNIVERSIDADES}

Respecto de los avances que podemos identificar, un indicador fundamental es el crecimiento de acciones vinculadas a la atención de las situaciones de violencia de género y su intervención con los protocolos de actuación. Desde el año 2013, y a través del activismo de docentes, investigadoras, no docentes, estudiantes y decanas, se comenzó un proceso de discusión y creación de protocolos y programas en las universidades para dar respuesta a las situaciones de discriminación y violencia de género vividas dentro de las instituciones. 
La incorporación de la agenda contra la violencia de género produjo, simultáneamente, el intercambio de experiencias y saberes en dos cuestiones concretas: cómo intervenir frente a los casos dentro de la universidad y el acompañamiento sobre el trabajo político para la creación y aprobación de los protocolos (Vazquez Laba y Rugna, 2017).

En el año 2014 la Universidad Nacional del Comahue ponía en práctica el primer protocolo de actuación contra la violencia de género, actualmente son 45 las universidades que cuentan con dicha herramienta, representando el 73,8\% del sistema universitario. Mientras que el 19,7\% de las instituciones universitarias restantes se encuentran diseñando y elaborando los proyectos de protocolos para ser aprobados próximamente. ${ }^{5}$ Desde el 2015 la cantidad de universidades que aprobaron sus protocolos aumentaron de forma continua, del 9\% en 2015 al casi 30\% en 2017.

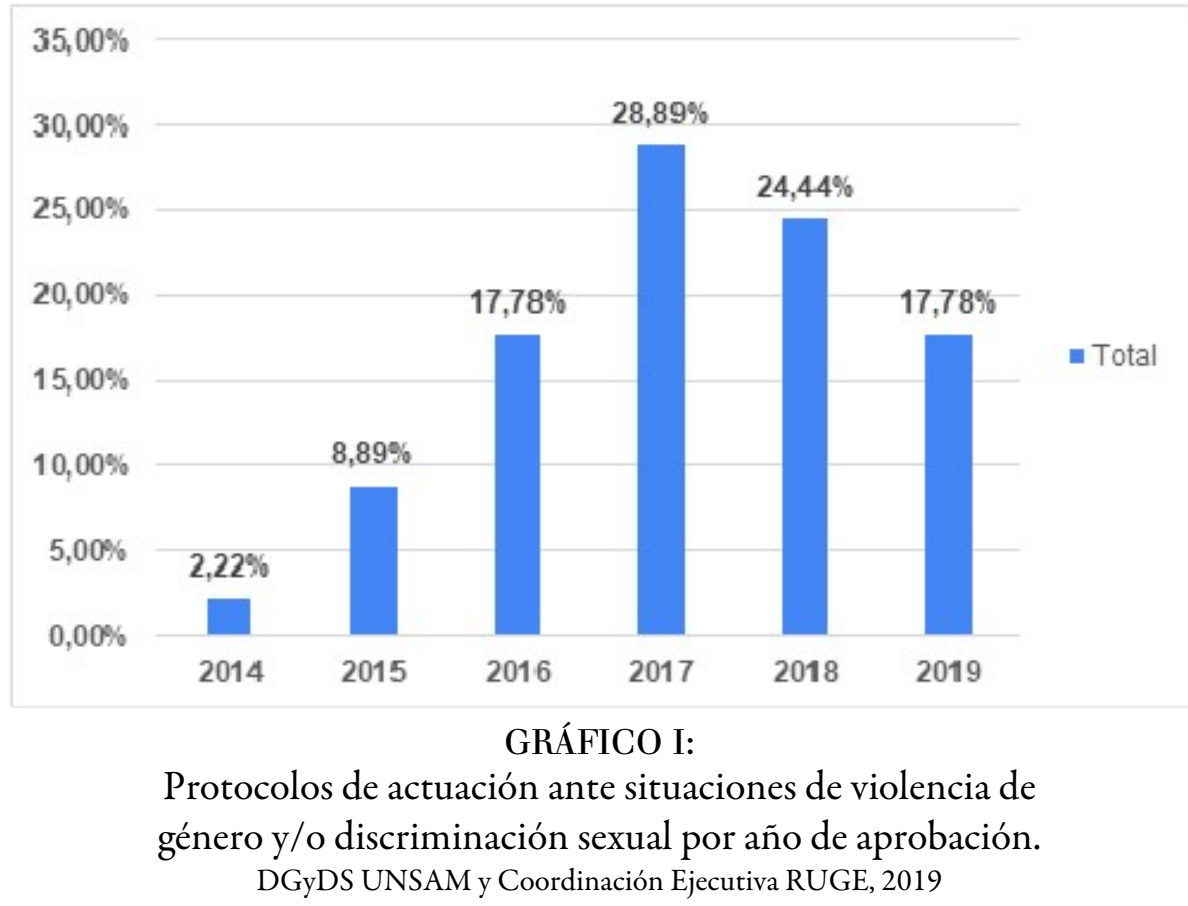

El aumento de 2017 y 2018 está relacionado con la acumulación de experiencia y trabajo político de las feministas universitarias sumado al proceso de movilizaciones que tuvo un claro auge en 2015 con el primer "ni una menos", luego el "paro internacional de mujeres" y en 2018 los "pañuelazos" a partir del debate en el Congreso de la Nación sobre la legalización y despenalización del aborto. ${ }^{6}$ Posteriormente, este año 2019, la adhesión del CIN a la ley 27.499, denominada "Ley Micaela", y su proceso posterior de legitimación por los Consejos Superiores terminó de impactar sobre las universidades que aún no tenían protocolos.

En un trabajo reciente (Vazquez Laba, 2019) se sostiene lo siguiente: "dentro del proceso de sensibilización y politización, el salto más importante ha sido instalar dentro de las universidades que la violencia de género es una falta grave que debe ser investigada, sancionada y erradicada" (pág. 4). En este sentido, este proceso en el cual se instala el problema de la violencia a partir del trabajo político por parte de las feministas universitarias en articulación con las redes del movimiento de mujeres y feminista a nivel regional y nacional es que se ha podido avanzar, también, en la transversalización del enfoque de género en las actividades de investigación y formación, pero, fundamentalmente, en las políticas institucionales. Este recorrido se ha dado de manera despareja en las universidades básicamente por las dificultades que supone la aplicación del protocolo y su alcance a toda la institución universitaria. La implementación de los dispositivos de atención y respuesta a las situaciones de violencia y discriminación sexual, lejos de operar como una pantalla de buenas prácticas, vino a visibilizar la necesidad de edificar espacios de género institucionalizados y que garanticen su aplicación para luego dar el paso a la transversalización institucional. 


\section{SEgUNDO PASO: INSTITUCIONALIZACIÓN DE LOS ESPACIOS DE GÉNERO}

Con el norte de desterrar la violencia de género, el androcentrismo y sexismo en las universidades, la praxis feminista universitaria da un segundo paso muy importante que viene siendo la modificación de las estructuras organizativas a partir de la incorporación de espacios de género y, por supuesto, nuevas lógicas de funcionamiento y producción de conocimiento (Vazquez Laba, 2019). Esto va en línea con algunos planteamientos de algunos organismos internacionales como la UNESCO que proponen que la dimensión de género es un indicador fundamental para medir la calidad educativa.

La RUGE es un actor fundamental para este proceso de jerarquización y profesionalización de las políticas de género en las universidades. Tal es así, que los datos evidencian casi el $60 \%$ de las universidades tienen un espacio institucionalizado de género, de las cuales el 55,2 \% tiene rango de "Programa" lo que da cuenta de un proceso incipiente, pero con muestras de un crecimiento y evolución ya que, como se observa en el siguiente gráfico, se vienen creando Secretarías, Sub-secretarías y Direcciones como espacios de mayor jerarquía.

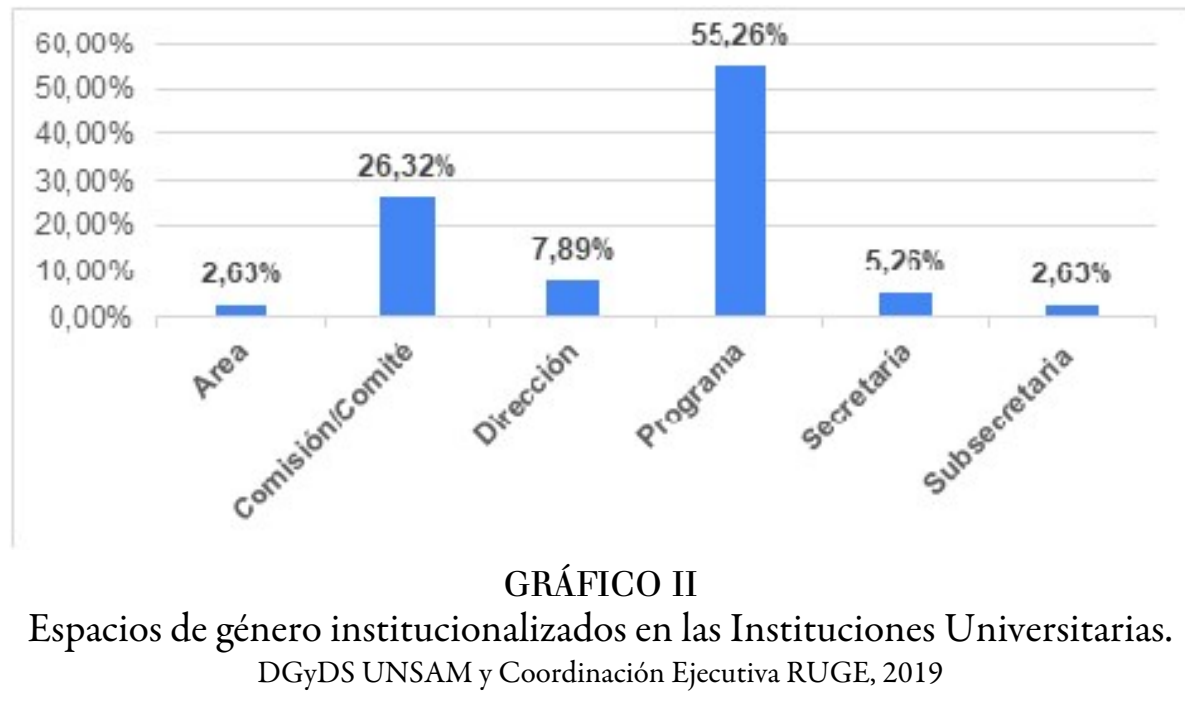

Los siguientes dos gráficos son importantes porque evidencian los recursos económicos que destinan las universidades a las políticas de género. Si bien sabemos que la existencia de los espacios institucionalizados no garantiza naturalmente recursos financieros para su desarrollo, por el contrario, se inserta en la disputa política por los recursos dentro de las instituciones universitarias. Teniendo en cuenta este dato, si observamos que el $25 \%$ de los espacios cuenta con presupuesto propio y que el $54 \%$ tiene asegurado al menos una persona trabajando dentro del espacio de género, habla no de debilidad sino, por el contrario, de la fuerza y el impacto de la praxis feminista y sus articulaciones y alianzas (Vazquez Laba, 2019). Si seguimos analizando, el 47,5\% de ese valor cuenta entre 2 a 5 personas para el trabajo institucional de género, es decir, una estructura de gestión bastante consolidada. 


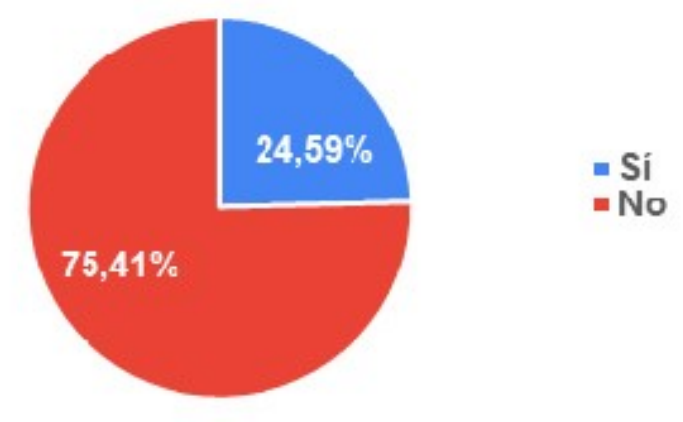

GRÁFICO III

Espacios de género que cuentan con presupuesto propio en las instituciones universitarias. Fuente: DGyDS UNSAM y Coordinación Ejecutiva RUGE, 2019

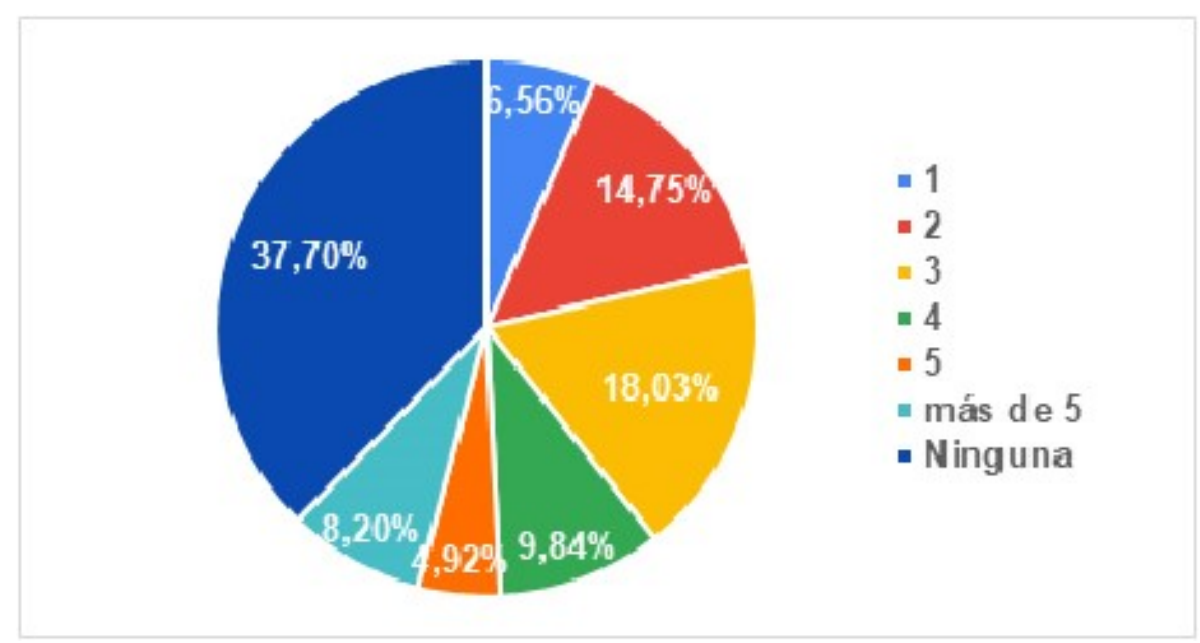

GRÁFICO IV

Cantidad de personas contratadas en los espacios de género de las instituciones universitarias. DGyDS UNSAM y Coordinación Ejecutiva RUGE, 2019

En definitiva, los recursos económicos son un tema sensible y de disputa de poder en todas las instituciones y a lo que se le suma que se disputa desde una temática que no es de la naturaleza universitaria, con lo cual, que el 75,4\% de las universidades todavía no tengan presupuesto propio nos obliga a revisar las estrategias colectivas y de demanda hacia el Estado nacional para generar condiciones de igualdad para garantizar dicha política en todo el sistema universitario.

\section{Tercer Paso: capacitaciones en el marco de la "Ley Micaela”}

En otro trabajo (Vazquez Laba, 2019) se plantea que los avances en materia de género dentro del sistema universitario, obliga a pensarlos en términos de emancipación, pero ya no como se entendía por el feminismo de los ' 60 y ' 70 sino, como un nuevo orden que desarma las desigualdades en el marco de una "reevaluación de las instituciones desde una perspectiva emancipadora" (Bolstanski y Fraser, 2019: 69), y que asegura el estado de derecho.

Como ya lo hemos mencionado, dentro el recorrido de politización del género en las universidades uno de los saltos más importante fue instalar la violencia de género como una falta que habilite medidas administrativas o medidas pedagógicas para su resolución. Lo que significa que las instituciones debieron 
abordar el problema de la desigualdad de género y la protección de los sujetos subalternizados por esa desigualdad.

La presentación del proyecto de adhesión a la "Ley Micaela" 7 en el plenario de Rectores del Consejo Interuniversitario Nacional en el mes de mayo, fue otro paso decisivo de la acción colectiva y estratégica feminista. Presentado por la Rectora coordinadora de la RUGE, Sandra Torlucci y acompañado por las palabras de "Yuyo" García, el papá de Micaela, el proyecto fue aprobado por unanimidad.

Como observamos en el siguiente gráfico, desde la adhesión a la ley hasta la actualidad el 81,9\% de las universidades han logrado su adhesión.

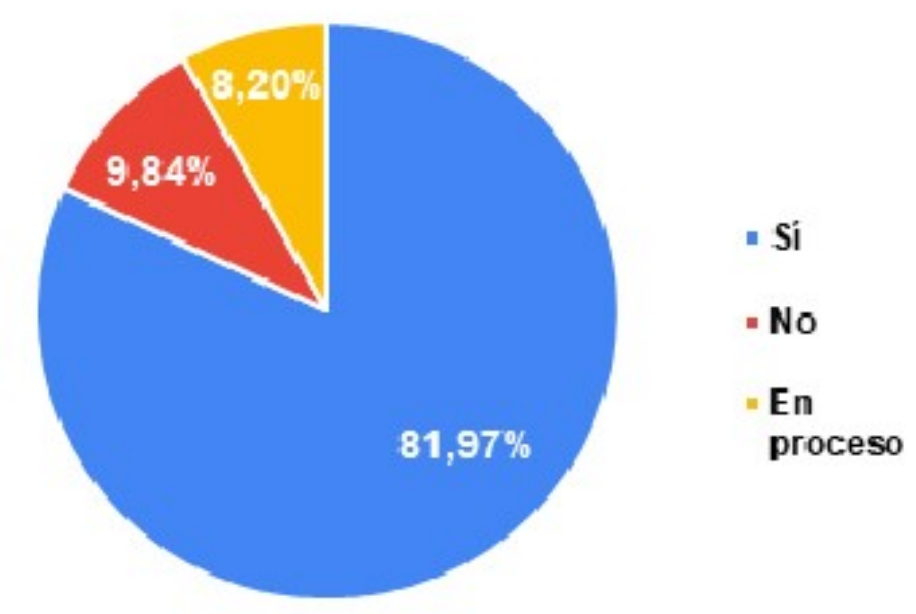

GRAFICO V

Instituciones Universitarias que adhirieron a la "Ley Micaela." DGyDS UNSAM y Coordinación Ejecutiva RUGE, 2019.

Del total de instituciones universitarias, el 47,5\% comenzó con las capacitaciones; $31 \%$ con las autoridades; $27 \%$ al claustro no docente; y el $21 \%$ al claustro docente y estudiantil. Como se observa por los datos, es inminente el proceso acelerado de formación en el marco de la ley.

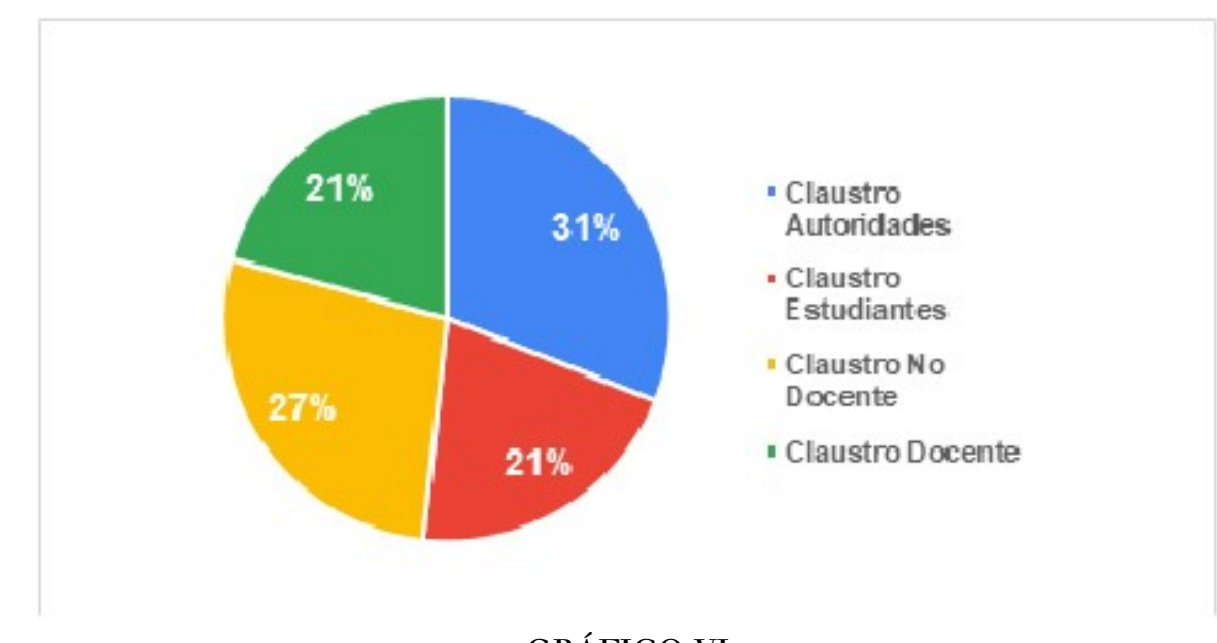

GRÁFICO VI

Capacitaciones en el marco de la "Ley Micaela" realizadas según claustro DGyDS UNSAM y Coordinación Ejecutiva RUGE, 2019. 
Un dato cualitativo y que complementa la interpretación del estudio de la RUGE, da cuenta que el proceso de capacitaciones se está llevando a cabo también desde la solidaridad feminista entre las universidades con mayor trayectoria en esta segunda generación de las políticas de género. La Universidad Nacional de San Martín, la Universidad Nacional de Rosario, la Facultad de Filosofía y Letras de la Universidad Nacional de Buenos Aires son los equipos convocados para las capacitaciones en otras universidades. No obstante, los equipos de la Universidad Nacional de Avellaneda, la Universidad Nacional de la Plata, la Universidad Nacional de General Sarmiento han sido las pioneras en esta política formativa.

\section{Los PASOS QUE SIgUen. REFLEXIONES FINALES}

Las prácticas concretas de creación de protocolo, transversalización del enfoque de género y capacitaciones son las acciones que están estableciendo un nuevo orden institucional y social en las universidades. Este nuevo orden produce, además, nuevos marcos de interpretación de la realidad social en el cual la justicia de género empieza a formar parte de los discursos y prácticas institucionales dejando de a poco de lado su mirada históricamente androcéntrica.

La "segunda reforma del '18 debe ser feminista", como se manifestó en un documento elaborado por la RUGE para el centenario de la Reforma: "Cualquier discusión programática de cara a los siguientes 100 años de nuestras universidades públicas no puede olvidar que una sociedad más justa e igualitaria es una sociedad que se piensa y se desea a sí misma libre de cualquier opresión de género, y proponiendo la ampliación de las pluralidades de los sujetos que la componen. Así -parafraseando las palabras finales del Manifiesto-, las feministas de las universidades, por intermedio de su Red, saludamos a los compañeros y las compañeras de la América toda y les incitamos a colaborar en la obra de libertad que inicia”.

En un proceso de feminización del sistema universitario, donde ya hay vasta producción científica sobre la importancia de la incorporación de los sujetos sexuados a los procesos institucionales para promover los derechos e igualdad, todavía hace falta avanzar en la paridad de participación de los órganos de gobierno de la universidad y en los espacios de toma de decisión. A la pregunta sobre la necesidad de incorporar mujeres y otras identidades sexuales y de género la respuesta es que una universidad gestionada con esta pluralidad y diversidad de miradas y experiencias asegura mayores niveles de democratización e igualdad.

\section{REFERENCIAS}

Boltanski, Luc y Fraser Nancy (2016). Dominación y emancipación. Buenos Aires, Capital intelectual.

Vazquez Laba, Vanesa (2019). "Políticas feministas en las universidades: revisando la idea de emancipación" en Jornadas de la Red Interdisciplinaria de Estudios de Género. Mesa 8: Pensamiento feminista desde el conurbano: universidad, investigación y política, UNTREF, octubre (mimeo).

Vazquez Laba, Vanesa y Rugna, Cecilia (2017). “Acción colectiva en torno a la agenda feminista sobre violencia de género en las Universidades Nacionales argentinas”. Boletin Cientifico Sapiens Reserch, Vol. 7, Bogotá.

\section{OTRAS FUENTES}

Pronunciamiento de la Red Universitaria de Género (RUGE) en el Centenario de la Reforma.

Pagina12 https://www.pagina12.com.ar/diario/suplementos/las12/13-10069-2015-10-02.html

Noticias UNSAM http://noticias.unsam.edu.ar/2019/04/22/ley-micaela-autoridades-de-universidades-del-cin-se-f ormaran-en-perspectiva-de-genero/

Noticias UNSAM http://noticias.unsam.edu.ar/2019/08/20/ley-micaela-en-la-unsam-hacia-una-comunidad-librede-violencia/ 


\section{Notas}

1 La Coordinación Ejecutiva está a cargo de Vanesa Vazquez Laba, Directora de la Dirección de Género y Diversidad Sexual de la Universidad Nacional de San Martín. En cuanto a la Comisión Ejecutiva, queda conformada de la siguiente manera: Universidad Nacional de San Martín y Universidad Nacional General Sarmiento en la coordinación del CPRES METROPOLITANO; la Universidad Nacional de La Plata en la coordinación del CPRES BONAERENSE; las universidades de Rosario y Río Cuarto en la coordinación del CPRES CENTRO; la Universidad Nacional de Cuyo en la coordinación del CPRES NUEVO CUYO; la Universidad Nacional de Jujuy en la coordinación del CPRES NOA; la Universidad Nacional de Misiones en la coordinación del CPRES NEA; y la Universidad Nacional de la Patagonia Austral en la coordinación del CPRES SUR.

2 Los Rectores en el Plenario del CIN adhirieron a la ley Micaela a través de un acuerdo plenario que establece en el artículo 2 lo siguiente: "Invitar a las instituciones universitarias públicas del Consejo Inter universitario Nacional a la organización de la capacitación obligatoria en materia de género y violencia contra las mujeres prevista en la Ley No 2749"Ley Micaela”; para sus autoridades superiores, docentes, no docentes y estudiantes" (Acuerdo Plenario del CINN $\left.{ }^{\circ} 1076 / 19\right)$.

3 Cada universidad designa dos representantes, titular y suplente, elegidas nominalmente por la autoridad máxima de la universidad para representar a la institución en la Red. En los casos que aún no se cuentan con representantes designadas, la encuesta ha sido respondida por referentes institucionales de la universidad que forman parte de las áreas de género.

4 El CIN está compuesto por 66 instituciones universitarias: 57 nacionales, 4 provinciales y 5 institutos universitarios. El estudio relevó información de 61 instituciones universitarias: 56 universidades nacionales, 4 provinciales y el Instituto Patagónico de las Artes; dejando afuera 4 institutos universitarios y la Universidad Nacional Scalabrini Ortiz por encontrarse en proceso de normalización.

5 La Secretaría de Políticas Universitarias lanzó en junio del corriente año una convocatoria en la que la RUGE colaboró, con el objetivo de financiar la elaboración o el fortalecimiento de los protocolos para atención en situaciones de discriminación y/o violencia de género en las universidades.

6 La articulación entre el movimiento feminista y las universidades, con las feministas universitarias como actrices fundamentales de la institucionalización de la perspectiva de género en el sistema universitario, se vio atravesado y fortalecido de forma determinante por la masificación del movimiento feminista y por la creciente visibilización de las principales demandas del movimiento. El primer "Ni Una Menos” en el año 2015 instalo la problemática de la violencia de género como un problema público, así como los medios de comunicación hegemónicos comenzaron a hablar de femicidios, las universidades comenzaron a recorrer sus propios procesos de problematización y respuesta a las situaciones de violencia de género en el ámbito universitario.

7 En enero de 2019 se sancionó la Ley 27.499, que lleva el nombre de Micaela García, víctima de un brutal femicidio cometido en Gualeguay (Entre Ríos). Néstor Yuyo García, el papá de Micaela — quien además fue decano de la Facultad Regional de la UTN-, impulsó este proyecto pedagógico en reconocimiento a la militancia por los derechos de las mujeres y de lxs más vulnerables que su hija llevaba adelante desde el Movimiento Evita. 